\title{
Blood Pressure over Height Ratios: Simple and Accurate Method of Detecting Elevated Blood Pressure in Children
}

\author{
Ovidiu Galescu, ${ }^{1}$ Minu George, ${ }^{1}$ Sudhakar Basetty, ${ }^{1}$ Iuliana Predescu, ${ }^{1}$ \\ Anil Mongia, ${ }^{2}$ Svetlana Ten, ${ }^{1}$ and Amrit Bhangoo ${ }^{1}$ \\ ${ }^{1}$ Division of Pediatric Endocrinology Infants and Children's Hospital of Brooklyn at Maimonides \& Children and Hospital at
Downstate, State University of New York, Brooklyn, NY 11219, USA
${ }^{2}$ Division of Pediatric Nephrology, Children's Hospital at Downstate, State University of New York, Brooklyn, NY 11219, USA
}

Correspondence should be addressed to Amrit Bhangoo, amrit.bhangoo@downstate.edu

Received 14 October 2011; Revised 19 January 2012; Accepted 9 February 2012

Academic Editor: Therese C. Jungraithmayr

Copyright (C) 2012 Ovidiu Galescu et al. This is an open access article distributed under the Creative Commons Attribution License, which permits unrestricted use, distribution, and reproduction in any medium, provided the original work is properly cited.

\begin{abstract}
Background. Blood pressure (BP) percentiles in childhood are assessed according to age, gender, and height. Objective. To create a simple BP/height ratio for both systolic BP (SBP) and diastolic BP (DBP). To study the relationship between BP/height ratios and corresponding BP percentiles in children. Methods. We analyzed data on height and BP from 2006-2007 NHANES data. BP percentiles were calculated for 3775 children. Receiver-operating characteristic (ROC) curve analyses were performed to calculate sensitivity and specificity of $\mathrm{BP} /$ height ratios as diagnostic tests for elevated BP $(>90 \%)$. Correlation analysis was performed between $\mathrm{BP}$ percentiles and $\mathrm{BP} /$ height ratios. Results. The average age was $12.54 \pm 2.67$ years. $\mathrm{SBP} /$ height and $\mathrm{DBP} / \mathrm{height} \mathrm{ratios}$ strongly correlated with SBP \& DBP percentiles in both boys $\left(P<0.001, R^{2}=0.85, R^{2}=0.86\right)$ and girls $\left(P<0.001, R^{2}=0.85\right.$, $\left.R^{2}=0.90\right)$. The cutoffs of SBP/height and DBP/height ratios in boys were $\geq 0.75$ and $\geq 0.46$, respectively; in girls the ratios were $\geq 0.75$ and $\geq 0.48$, respectively with sensitivity and specificity in range of $83-100 \%$. Conclusion. BP/height ratios are simple with high sensitivity and specificity to detect elevated BP in children. These ratios can be easily used in routine medical care of children.
\end{abstract}

\section{Introduction}

Hypertension in children and adolescents is defined as systolic BP (SBP) and/or diastolic BP (DBP) above the 95th percentile. BP between the 90th and 95th percentile is designated "high normal" or "prehypertensive" [1]. Currently the available data to quantify hypertension in children is in the form of a table reference matched by sex, age, and height percentile that identifies the BP as 50th, 90th, 95th, and 99th percentiles [2].

In recent years the prevalence of hypertension in the pediatric population in the United States has been increasing [3]. Children and adolescents with primary hypertension are frequently overweight or obese. The prevalence of hypertension increases progressively with increasing body mass index (BMI), and hypertension is detectable in over $30 \%$ of overweight children with BMI above 95 th percentile [4]. The increase in the prevalence of childhood obesity and its strong association with hypertension make both prehypertension and hypertension a major health problem in the young [5].

Hypertension in childhood and adolescence can lead to hypertension as adults $[6,7]$. Although hypertension in childhood has been thought of as an independent risk factor for CVD (cardiovascular vascular disease), there are several studies demonstrating the association of hypertension with other risk factors such as hypercholesterolemia, hypertriglyceridemia, low HDL cholesterol, truncal (central) obesity, hyperinsulinemia, and metabolic syndrome $[8,9]$.

The criteria for diagnosis of hypertension in children and adolescents can be complex and challenging to apply in clinical practice because of age-, gender-, and height-specific reference values [10]. Both hypertension and prehypertension though prevalent and increasing in incidence remain widely undiagnosed [11]. In $2010 \mathrm{Lu}$ et al. evaluated, for the first time, the feasibility and accuracy of the systolic 


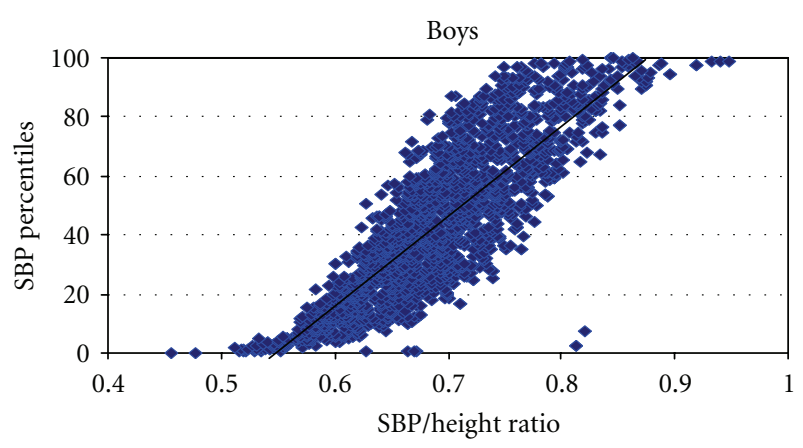

Figure 1: The graph depicts the strong correlation of systolic blood pressure percentiles (SBP Percentiles), on the $Y$ Axis and the systolic blood pressure over height ratio (SBP/height ratio) on the $X$ Axis in boys $P<0.001, R^{2}=0.85$, SBP: systolic blood pressure.

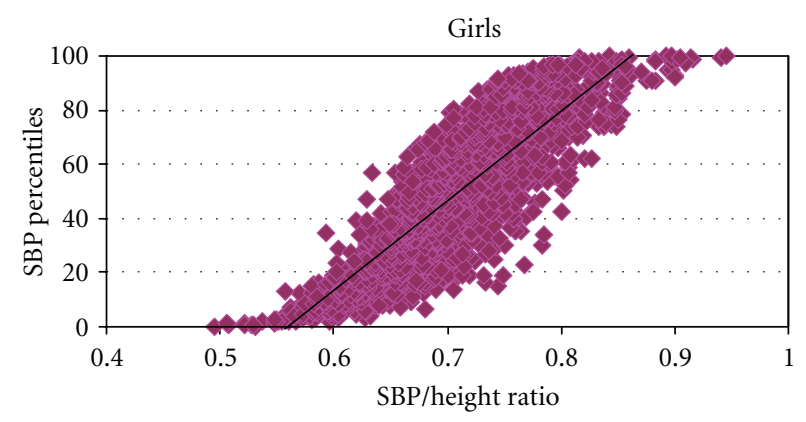

FIGURE 2: The graph depicts the strong correlation of systolic blood pressure percentiles (SBP Percentiles), on the $Y$ Axis and the systolic blood pressure over Height ratio (SBP/height ratio) on the $X$ Axis in girls $P<0.001, R^{2}=0.80$. SBP: Systolic Blood Pressure.

blood pressure-to-height ratio ( $\mathrm{SBP} /$ height ratio) and the diastolic blood pressure-to-height ratio (DBP/height ratio) and proposed the optimal thresholds of SBP/height ratio and DBP/height ratio for identifying hypertension in Chinese adolescents. They concluded that the $\mathrm{BP} /$ height ratios are simple, inexpensive, and accurate indices for identification of hypertension in adolescents [12]. Subsequently BP/height ratios and cutoffs were also proposed for Italian and Nigerian adolescents $[13,14]$.

In order to validate the $\mathrm{BP} /$ height ratios in US children, similar studies needed to be repeated and studied further. Therefore, this formed the basis of hypothesis of our study. In this study we tested that simple $\mathrm{BP} /$ height ratios can be used as an accurate clinical tool to identify children and adolescents at risk for hypertension. For this purpose we have reported the simplified ratios of $\mathrm{BP} /$ height as an accurate detection method of elevated BP. We also report the correlation of $\mathrm{BP} /$ height ratios with commonly used $\mathrm{BP}$ percentiles.

\section{Materials and Methods}

2.1. Data Collection. We analyzed data on 3775 children from NHANES 2007-2008 (National Health and Nutrition Examination Survey). Data on age, gender, ethnicity, height, weight, and waist circumference was analyzed [13]. Systolic blood pressure (SBP) and diastolic blood pressure (DBP), were reported to be measured by a standard protocol used by NHANES [14]. Body mass index (BMI) was calculated using the standard formula. BP percentiles were calculated according to the standard formula provided in the following referenced manuscript [2].

2.2. Statistical Analysis. Receiver-operating characteristic (ROC) curve analyses were performed to calculate sensitivity and specificity of $\mathrm{SBP} /$ height and $\mathrm{DBP} /$ height ratios as diagnostic tests for elevated, that is, $>90$ th percentile of SBP and DBP, respectively. Correlation analysis was performed between SBP percentile and SBP/height ratio. Similar correlation was studied for DBP percentiles and DBP/height ratio. ROC analyses and curve generation were performed using Analyse-it Method Evaluation edition. For data analysis and interpretation we used SPSS v.19.

\section{Results}

Data was generated from 1982 boys aged $12.5 \pm 2.6$ years old and 1783 girls aged $12.5 \pm 2.6$ years old. The distribution by ethnicity as defined in the NHANES Demographics Variable List was accessed. The various ethnicities were as follows: 1069: Mexican Americans, 383: other Hispanics, 1083: nonHispanic Whites, 1047: non-Hispanic Blacks, and 183-other Races. The BMI percentile in girls from all ethnic groups was $67 \pm 29$ (Mean \pm SD). Approximately 23 percent of girls had BMI greater than 95th percentile. The BMI percentile in boys from all ethnic groups was $66 \pm 30$ (Mean \pm SD). The proportion of boys with BMI greater than 95th percentile was approximately 24 percent. The proportion of overweight girls and boys with BP reading above 90th percentile was $11 \%$ and $10 \%$ respectively. The proportion of BP above 90 th percentile in all girls and boys was $6.3 \%$ and $5.2 \%$, respectively.

$\mathrm{SBP} /$ height ratio strongly correlated with SBP percentiles in both boys (Figure 1; $P<0.001, R^{2}=0.85$ ) and girls (Figure 2; $P<0.001, R^{2}=0.85$ ). Similar results were obtained for DBP/height ratio and DBP percentiles: boys' $P<0.001, R^{2}=0.86$ and girls' $P<0.001, R^{2}=0.90$. ROC analysis showed a very steep progression of sensitivity and specificity above certain cutoff values. The cutoffs of elevated $\mathrm{SBP} /$ height and $\mathrm{DBP} /$ height ratios in boys were $\geq 0.75$ and $\geq 0.46$; in girls the ratios were $\geq 0.75$ and $\geq 0.48$, respectively. Above the cutoff values of the ratios the sensitivity and specificity of detection of elevated BP (greater than 90th percentile) was very high as shown in (Table 1). There was no significant difference of $\mathrm{BP} /$ height ratios in various ethnicities as demonstrated by an inter-group weighted $t$ test (data not shown).

The accuracy of this determination of ratios above the 95th percentile is diminished by the low number of children. In this cohort there were only 50 boys $(2.6 \%)$ who had SBP percentiles above 95th percentile and only $6(0.3 \%)$ had DBP above this range. Similarly 46 girls $(2.6 \%)$ had SBP percentiles above 95 th percentile and only $7(0.4 \%)$ had DBP percentiles in the hypertensive range. 
TABLE 1: ROC analysis of SBP and DBP in boys and girls. Area under the curve (AUC) and corresponding $P$ values are depicted with the associated confidence intervals for the calculated cutoff values of the $\mathrm{BP} /$ height ratios greater than 90th percentile. Correlation of the $\mathrm{BP} /$ height ratios with their respective $\mathrm{BP}$ for each cutoff value is quantified by sensitivity and specificity percentages.

\begin{tabular}{lccccccc}
\hline & Cutoff BP/height ratios & AUC & $P$ value & CI & $N$ & Sensitivity (\%) & Specificity (\%) \\
\hline Boys & & & & & & & \\
SBP $>90 \%$ & $\geq 0.75$ & 0.95 & $<0.0001$ & $0.94-0.96$ & 1979 & 95 & 93 \\
DBP $>90 \%$ & $\geq 0.46$ & 0.95 & $<0.0001$ & $0.87-1$ & 1972 & 91.5 \\
\hline Girls & & & & & & & \\
SBP $>90 \%$ & $\geq 0.75$ & 0.96 & $<0.0001$ & $0.94-0.97$ & 1765 & 97.2 & 83.3 \\
DBP $>90 \%$ & $\geq 0.48$ & 0.99 & $<0.0001$ & $0.98-0.99$ & 1762 & 100 & 95.5 \\
\hline
\end{tabular}

SBP: systolic blood pressure, DBP: diastolic blood pressure, AUC: area under the curve, CI: confidence interval, and N: number of children in category.

\section{Discussion}

Hypertension in the pediatric and adolescent population may remain undiagnosed. Although the BP charts for age, gender and height are readily available, they are generally not used in a busy pediatric practice. The diagnosis of hypertension may be missed in some children [10]. In 2008 Cunningham reviewed data on 14,187 children and adolescents. Overall, 507 children fulfilled the criteria for hypertension, of whom 74\% were undiagnosed. Prehypertension was documented in 485 individuals, but $89 \%$ had not been previously diagnosed. [11] The authors concluded that the current practice of identifying the BP percentiles from lengthy tables can be cumbersome, ineffective, and can often miss the diagnosis of hypertension in children, which can become detrimental to their long-term health [11].

In this study we have shown that $\mathrm{BP} /$ height ratios are simple and accurate method of diagnosing elevated blood pressure. Our findings are in complete agreement with the preceding studies on this matter [12-14]. The authors in that study clearly demonstrated the feasibility and accuracy of the $\mathrm{SBP} /$ height and DBP/height ratios in their cohort of Chinese, Italian, and Nigerian adolescents [12-14]. In our study the cutoff for SBP/height ratio for both boys and girls is 0.75 . The cutoffs for DBP/height ratio in boys and girls are 0.46 and 0.48 , respectively (Table 1 ). The sensitivities and specificities of these ratios are from $83 \%$ to $100 \%$ in identifying elevated $\mathrm{BP}$ above the 90th percentile. Our study is the first to report the $\mathrm{BP} /$ height ratio for prehypertension, which can be used as a reliable tool not only for diagnosis but also screening for high $\mathrm{BP}$ in ambulatory care setting in children. Also, there were no significant differences of $\mathrm{BP} /$ height ratios in various ethnicities as demonstrated by intergroup weighted $t$ tests. Therefore, we have proven for the first time that these ratios can be used universally in most ethnic groups represented in USA In addition, we have shown that SBP/height and $\mathrm{DBP} /$ height ratios strongly correlated with their respective SBP and DBP percentiles in both boys and girls (Figures 1 and 2).

Our study has a large cohort of subjects derived from the NHANES database. Hence the ROC analysis for the calculated cutoffs generates good power and validation (Table 1). In this cohort the incidence of BP above 95th percentile was low (below $3 \%$ in both boys and girls). Therefore it is our belief that more studies need to be done with looking at a larger cohort of hypertensive children to assess the reliability and reproducibility of the $\mathrm{BP} /$ Height ratios. Although NHANES data focuses on US children and adolescents without any particular pathology, the large numbers of subjects in this study make it possible to extrapolate the results at a population level. Also this study is based on cross-sectional data and does not imply target organ damage, which would need standard diagnostic techniques such as echocardiogram.

\section{Conclusions}

$\mathrm{BP} /$ height ratio is a simple, excellent screening and diagnostic tool with high sensitivity and specificity to detect elevated $\mathrm{BP}$. The $\mathrm{BP} /$ height ratio can be easily used in routine medical care of children. The BP/height ratios also correlated with the corresponding BP percentiles in both genders. More studies are needed in patients with proven hypertension to validate the use of this proposed $\mathrm{BP} /$ height ratio in conditions with severe elevations of blood pressure.

\section{Abbreviations}

CDC: $\quad$ Centers for Disease Control and Prevention

NHANES: National Health and Nutrition Examination Survey

ROC: Receiver-operating characteristic

CVD: Cardiovascular vascular disease.

\section{References}

[1] A. V. Chobanian, G. L. Bakris, H. R. Black et al., "Seventh report of the Joint National Committee on Prevention, Detection, Evaluation, and Treatment of High Blood Pressure," Hypertension, vol. 42, no. 6, pp. 1206-1252, 2003.

[2] B. Falkner, S. R. Daniels, J. T. Flynn et al., "The fourth report on the diagnosis, evaluation, and treatment of high blood pressure in children and adolescents," Pediatrics, vol. 114, supplement 2, pp. 555-576, 2004.

[3] J. M. Sorof, D. Lai, J. Turner, T. Poffenbarger, and R. J. Portman, "Overweight, ethnicity, and the prevalence of hypertension in school-aged children," Pediatrics, vol. 113, no. 3, part 1, pp. 475-482, 2004.

[4] J. Sorof and S. Daniels, "Obesity hypertension in children: a problem of epidemic proportions," Hypertension, vol. 40, no. 4, pp. 441-447, 2002. 
[5] C. L. Ogden, K. M. Flegal, M. D. Carroll, and C. L. Johnson, "Prevalence and trends in overweight among US children and adolescents, 1999-2000," Journal of the American Medical Association, vol. 288, no. 14, pp. 1728-1732, 2002.

[6] R. M. Lauer and W. R. Clarke, "Childhood risk factors for high adult blood pressure: the Muscatine study," Pediatrics, vol. 84, no. 4, pp. 633-641, 1989.

[7] S. G. Kiessling, K. K. McClanahan, and H. A. Omar, "Obesity, hypertension, and mental health evaluation in adolescents: a comprehensive approach," International Journal of Adolescent Medicine and Health, vol. 20, no. 1, pp. 5-15, 2008.

[8] S. Cook, M. Weitzman, P. Auinger, M. Nguyen, and W. H. Dietz, "Prevalence of a metabolic syndrome phenotype in adolescents: findings from the Third National Health and Nutrition Examination Survey, 1988-1994," Archives of Pediatrics and Adolescent Medicine, vol. 157, no. 8, pp. 821827, 2003.

[9] C. L. Williams, L. L. Hayman, S. R. Daniels et al., "Cardiovascular health in childhood: a statement for health professionals from the Committee on Atherosclerosis, Hypertension, and Obesity in the Young (AHOY) of the Council on Cardiovascular Disease in the Young, American Heart Association," Circulation, vol. 106, no. 1, pp. 143-160, 2002.

[10] M. L. Hansen, P. W. Gunn, and D. C. Kaelber, "Underdiagnosis of hypertension in children and adolescents," Journal of the American Medical Association, vol. 298, no. 8, pp. 874-879, 2007.

[11] R. J. Cunningham III, "Is pediatric hypertension underdiagnosed?" Nature Clinical Practice. Cardiovascular Medicine, vol. 5, no. 3, pp. 128-129, 2008.

[12] Q. Lu, C. M. Ma, F. Z. Yin, B. W. Liu, D. H. Lou, and X. L. Liu, "How to simplify the diagnostic criteria of hypertension in adolescents," Journal of Human Hypertension, vol. 25, pp. 159-163, 2011.

[13] F. Rabbia, I. Rabbone, S. Totaro et al., "Evaluation of blood pressure/height ratio as an index to simplify diagnostic criteria of hypertension in Caucasian adolescents," Journal of Human Hypertension, vol. 25, no. 10, pp. 623-624, 2011.

[14] C. E. Ejike, "Blood pressure to height ratios as simple, sensitive and specific diagnostic tools for adolescent (pre)hypertension in Nigeria," Italian Journal of Pediatrics, vol. 37, article 30, 2011. 


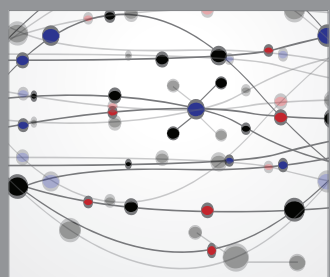

The Scientific World Journal
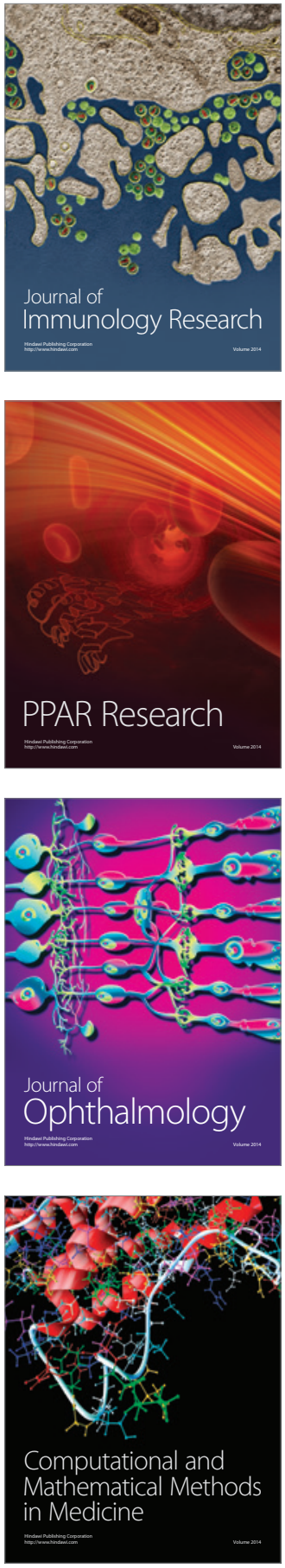

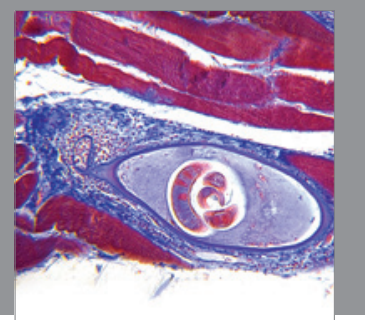

Gastroenterology

Research and Practice
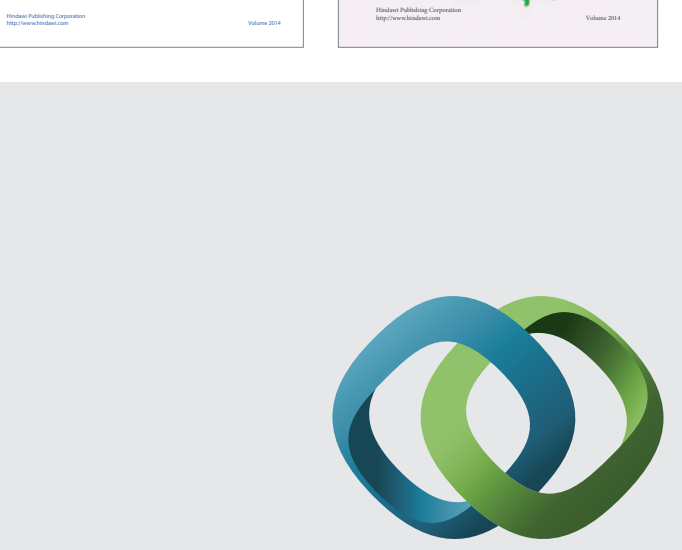

\section{Hindawi}

Submit your manuscripts at

http://www.hindawi.com
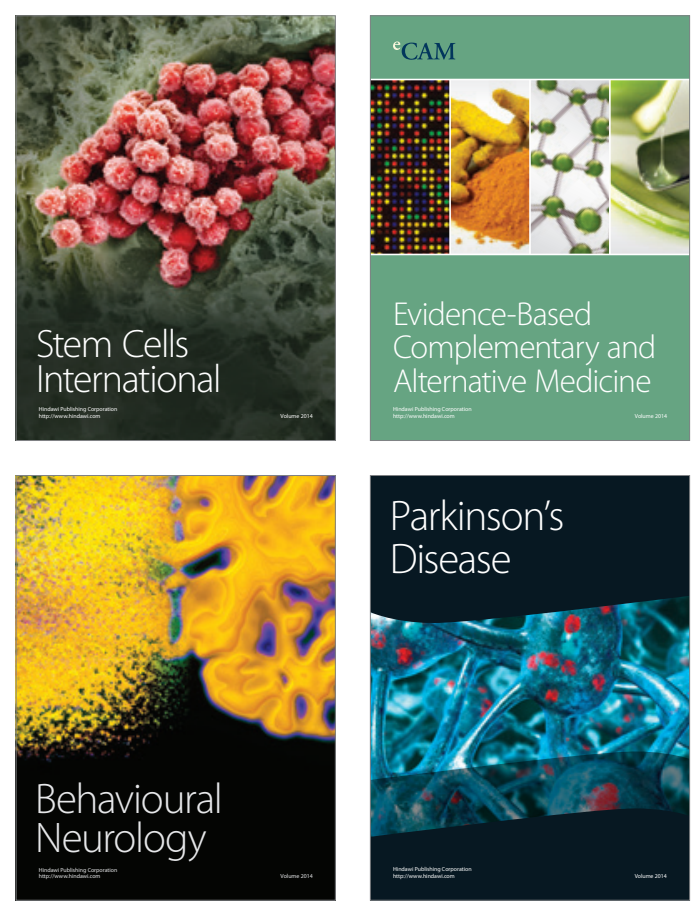

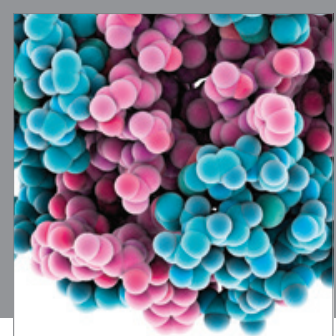

Journal of
Diabetes Research

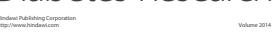

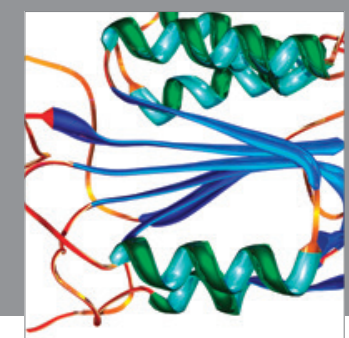

Disease Markers
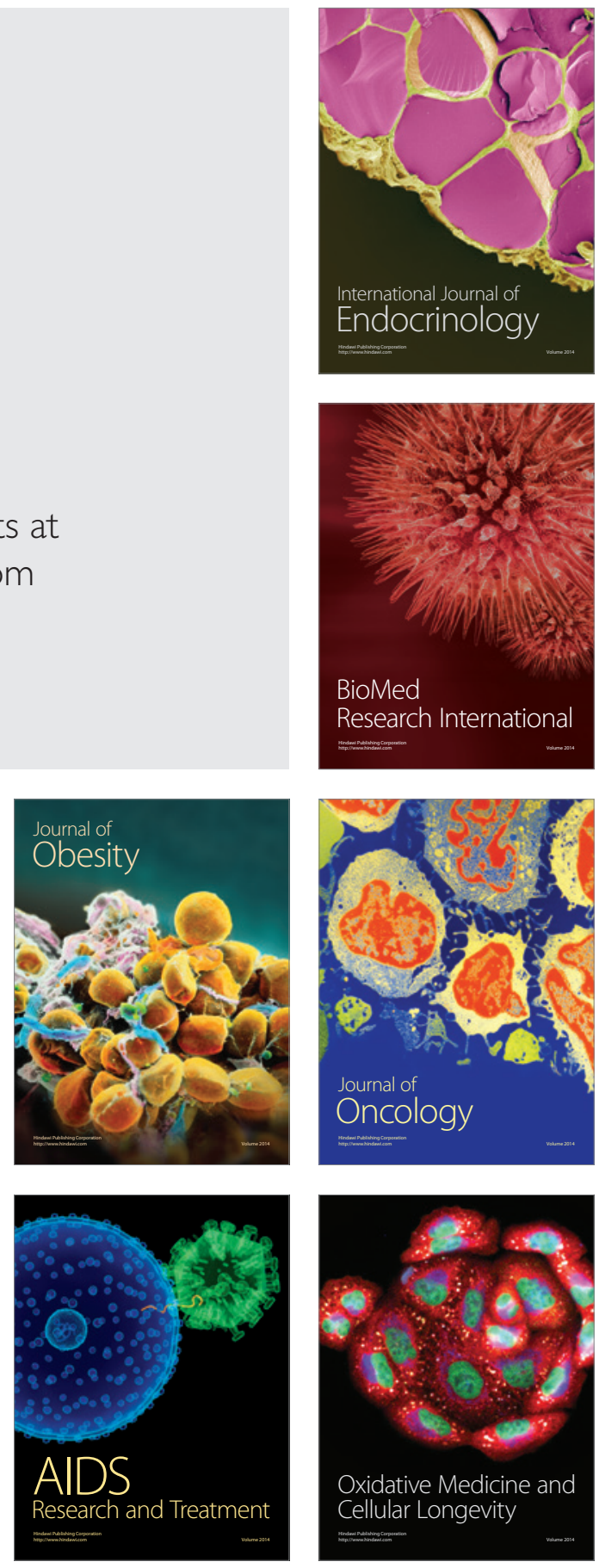